\title{
Autour du bûcher de Jeanne
}

\section{Françoise Michaud-Fréjaville}

\section{OpenEdition}

Journals

Édition électronique

URL : https://journals.openedition.org/crm/2473

DOI : $10.4000 / \mathrm{crm} .2473$

ISSN : 1955-2424

\section{Éditeur}

Honoré Champion

\section{Édition imprimée}

Date de publication : 15 janvier 1997

Pagination : 131-141

ISSN : 1272-9752

\section{Référence électronique}

Françoise Michaud-Fréjaville, «Autour du bûcher de Jeanne », Cahiers de recherches médiévales [En ligne], 3 | 1997, mis en ligne le 06 août 2008, consulté le 15 décembre 2022. URL : http:// journals.openedition.org/crm/2473; DOI : https://doi.org/10.4000/crm.2473 


\section{Autour du bûcher de Jeanne}

«Tu ne laissera pas en vie la magicienne » (Exode, XXII, 17)

Abstract: Relying on the case of the heretics of Orleans (1022), it is possible to reconsider the testimonies of contemporary chronicles relating Joan of Arc's death. What did the penalty by fire mean for its upholders and its opponents?

La sentence nous vient du fond des âges: la crainte des maléfices est plus forte que la loi du décalogue. Au «tu ne tueras» pas (Exode, $\mathrm{XX}, 13$ ) répond: «quiconque frappe quelqu'un et cause sa mort, devra être mis à mort [...] si quelqu'un va jusqu'à assassiner son prochain, tu l'arracheras même de mon autel pour qu'il soit mis à mort » (Exode, XXI, 12-14).

L'ancien testament trouve un écho dans l'Épître de saint Jude : «Il s'est glissé parmi vous certains hommes qui depuis longtemps ont été marqués d'avance pour cette sentence; ces impies travestissent en débauche la grâce de notre Dieu et renient notre maître et seigneur, Jésus-Christ. Quant aux anges qui n'ont pas conservé leur primauté mais qui ont quitté leur propre demeure, c'est pour le jugement du grand jour qu'il les a gardés dans des liens éternels, au fonds de ténèbres. Ainsi Sodome et Gomorrhe et les villes voisines [...] sont-elles proposées en exemple, subissant la peine d'un feu éternel " (Jude, 5-7). Ceux qui se targuaient de prétendues révélations, de fausses visions furent assimilés à des magiciens suscitant des démons, pour ce que Norman Cohn appelait des«miracles pernicieux " $"$. Les gens convaincus d'hérésie ont très vite été considérés comme des êtres animés non pas seulement de leur propre volonté mais de celle des anges déchus. Il peut donc paraitre assez évident que le feu des villes maudites (Genèse, $\mathrm{XIX}, 23)$ dût être leur destin terrestre, préludant au brasier qui ne pourrait être éteint, au pardon impossible, à la Géhenne éternelle.

Saint Jean fournit la référence fondamentale qui a nourri ensuite les formulaires des juges de l'Inquisition: "si quelqu'un ne demeure pas en moi, on le jette dehors comme le sarment et il se dessèche: puis on les ramasse [les sarments] et on les jette au feu, et ils brûlent ". (Jean, XV, 6) Ainsi, "comme membre pourri, nous t'avons déboutée et rejettée " ${ }^{2}$ dirent à Jeanne ses juges, le 30 mai 1431, avant de la livrer aux soldats de Warwick et de se retirer sans assister à la mise à mort.

L'édit de persécution de Dioclétien (en 297) prévoyait cette peine du feu à l'encontre des Manichéens, accusés de collusion avec les Perses (qui cependant les pourchassaient également) : «Il est à craindre [...] qu'ils n'utilisent les moeurs

\footnotetext{
${ }^{1}$ N. Cohn, Démonolâtrie et sorcellerie au Moyen Age, fantasmes et réalités, Paris, 1982, p. 201 et suivantes.

'Procès de condamnation de Jeanne d'Arc. éd. P. Tisset avec la collaboration d'Yvonne Lanhers, Paris, Klincksieck, 1960, (Société de l'histoire de France, Fondation du département des Vosges), t. I, Texte, p. 413 : te tamquam membrum putridum [...] abscindimus.
}

Cahiers de Recherches Médiévales (XIIf-XV S.). 3. 1997 
abominables et les funestes lois des Perses pour tenter d'inoculer le venin de leur vilenie à des hommes d'une nature plus vertueuse, à l'honnête et paisible peuple romain et à notre univers tout entier [...]. Nous ordonnons qu'instigateurs et dirigeants soient soumis, eux et leurs abominables écrits, au châtiment le plus rigoureux en étant livrés aux flammes $n^{3}$. Non seulement les fidèles mais aussi les livres manichéens furent la proie des flammes

Les premiers hérétiques du Moyen Age à avoir été soumis à cette peine furent des chanoines d'Orléans et leurs disciples, en $1022^{4}$. Refusant d'être convaincus, après enquête et réunions contradictoires, dont une séance au moins fut publique, de renoncer à leurs erreurs, ils préférèrent ce qui êtait pour eux le martyre. Or beaucoup de gens depuis le IV siècle, à l'imitation de saint Augustin, baptisaient «manichéenne » toute déviation. On peut comprendre le choix du bûcher fait par Robert le Pieux et ses prélats et conseillers: ils se référaient à une triple tradition évangélique, patristique et romaine.

Il n'est pas indifférent de noter les termes qu'utilisèrent les histoires contemporaines pour rapporter les circonstances de ces morts affreuses. La preuve de la collusion des accusés avec les forces du mal est claire dans les déclarations que l'on prête aux déviants : le feu ne les touchera pas. Ainsi Raoul Glaber écrit : «ils se vantaient de n'avoir peur de rien, ils annonçaient qu'ils sortiraient du feu indemnes $»^{5}$. Les hérétiques, pour la plupart cultivés, connaissaient évidemment la parole d'Isaïe : «si tu as la foi, la flamme ne te brûlera pas » (Isaïe, 43, 2). Selon Adémar de Chabannes «ils prétendaient qu'ils sortiraient indemnes des flammes et c'est en riant qu'ils se laissèrent attacher au milieu du bûcher ${ }^{6}$. L'aide de Dieu - ou du Malin - ne se produisit pas, les condamnés souffrirent, reconnurent avoir été abusés par le démon ce qui justifiait évidemment la sentence du roi Robert. Mais comme ils étaient des clercs ils comprenaient qu'ils méritaient leur sort et que c'était l'entrée en damnation: «ils commençaient déjà à brûler plus fort, quand ils se mirent de toute la force de leur voix à crier au milieu du brasier qu'ils avaient été horriblement abusés par les artifices du démon [...] et qu'en châtiment du blasphème dont ils s'étaient rendus coupables, ils enduraient mille tourments en ce monde en attendant les tourments éternels qu'ils allaient subir dans l'autre » (R. Glaber). Les personnes présentes ne pouvaient plus intervenir pour adoucir la sentence, le pardon n'était plus de leur ressort: «les assistants poussés par la pitié et l'humanité, s'approchèrent pour arracher du moins au feu ceux qui n'étaient qu'à demi brûlés, mais ils n'y réussirent point " ( $R$. Glaber). La justice a été rendue par la disparition totale des mauvais: "ils furent totalement réduits en cendres et l'on ne retrouva même pas un débris de leurs os" (A. de Chabannes). L'autre récit rend un son

${ }^{3}$ F. Decret, Mani et la tradition manichéenne, Paris, 1974, pp.145-146.

${ }^{4}$ R.-H. Bautier, "L'hérésie d'Orléans et le mouvement intellectuel au début du XI siècle, documents et hypothèses", Actes du $95^{\circ}$ Cangrès national des Sociétés savantes, Reims, 1970, Paris, 1975, pp. 63-88. Et H. Taviani. "Le mariage dans l'hérésie de l'An mil, ", Annales ESC, 1977, n 6, pp. 1074-1089.

${ }^{5}$ Raoul Glaber, Histoires, éd. M. Prou, Paris,1886. livre III, VIII, pp. 74-81.

6 Adémar de Chabannes, Chroniques, éd. Chavannon. Paris, 1897, livre III, LIX, pp.184-185. 
semblable: « la flamme justicière achevait de consommer ces malheureux et les réduisit incontinent en cendres " ( $\mathrm{R}$. Glaber). Le roi avait présidé à un châtiment qui s'était voulu public, en vertu de la valeur exemplaire de la peine. Les historiens ne furent pourtant pas tout à fait d'accord sur les modalités pratiques. Selon nos deux principales sources, il est certain que le bûcher était un feu visible, cérémoniel, que les assistants le virent, l'entendirent crépiter, qu'ils purent mesurer le degré d'avancement de l'horrible agonie des chanoines, et même tenter de sauver ceux pour lesquels certains devaient éprouver de l'amitié, voire de l'affection ou ceux dont une contrition sincère aurait mérité la compassion. On pouvait s'approcher quoique, en raison de la nécessité de l'édification des assistants et des lecteurs, cela ne puisse pas servir à sauver les condamnés mais au contraire à prouver que la justice du roi avait rejoint la justice de Dieu. Un troisième récit, celui de la Vita de Gauzlin, abbé de Saint-Benoît, archevêque de Bourges et peut-être demi-frère de Robert, rapporte seulement que « le roi ordonna de les livrer au feu pour en donner possession au feu pour l'éternité ${ }^{7}$. Le jugement était ici sans le moindre appel : il n'y avait pas de témoins d'un repentir des hérétiques, pas de geste charitable puisqu'il ne pouvait qu'être inutile. Le roi est là aussi le lieutenant de Dieu sur terre, son jugement et les châtiments qu'il ordonne sont eschatologiques.

Rien ne s'arrête avec les cendres refroidies des intellectuels punis. L'élan donné par Robert le Pieux, bras séculier d'une église militante qui se sépare des rameaux morts ou gangrenés, traversa le Moyen Age : “ Depuis lors, partout où l'on a découvert des adeptes de ces croyances perverses, on les a livrés au même châtiment vengeur » (R. Glaber). Et immédiatement aussi la répression devint pour les pasteurs un cas de conscience qui ne se résolvait pas avec facilité. En 1024-1025 à Arras et Cambrai, la grave question de la répression de nouvelles déviations amena les évêques à tenter de persuader les déviants, plutôt qu'à les détruire. G Duby a montré, peut-être en plaçant un peu trop la seule sexualité au centre des débats, comment des définitions nouvelles des trois ordres de la société amenèrent Gérard de Cambrai à trouver un terrain d'accord, ou plutôt comment ce dernier réussit à amener les hérétiques à accepter des divisions de la société dans lesquelles ils pourraient s'insérer, avec leur «désir forcené d'étendre leur monachisme au peuple chrétien tout entier ${ }^{8}$. Cependant ailleurs, à Goslar en Allemagne, et à Reims surtout, le modèle orléanais de l'hérésie avait été suivi. tout comme sa répression par le feu avait été assez largement imitée. Aussi l'évêque de Châlons, dans une Champagne en proie aux hérétiques, chercha-t-il auprès de ses collègues des conseils pour guider son action. On a conservé la réponse de l'évêque de Liège, Wason. Elle est le contrepoint parfait de l'attitude des rois et évêques capétiens : "Nous sommes invités non à donner la mort mais plutôt, avec le concours de Dieu, à donner la vie » ${ }^{9}$.

La maturation fut lente de la mise en place des procédures de lutte contre les hérésies. L'Inquisition finit au XIIe siècle par trouver une sorte de tragique

\footnotetext{
${ }^{7}$ André de Fleury, Vie de l'abbé Gauzlin. éd. R.-H. Bautier. Paris, 1969, pp. 97-99.

${ }^{8} \mathrm{G}$. Duby, Les trois ordres ou l'imaginaire du féodalisme. Paris, 1978, pp. 161-182.

9 Abbé Migne, Pat.Lat., t. CXLII, col 751.
} 
compromis entre la nécessité d'éradiquer les partisans de déviations considérées comme mortelles pour la société et une tradition de cléricature désarmée. Certes, l'Église qui horret a sanguine a dévolu au bras séculier ses condamnés mais le feu, non sanglant, permit aussi un respect, au moins formel et non sans une certaine forme d'hypocrisie, de la répulsion à verser le sang hautement affirmée par les clercs $^{10}$.

Le bûcher de Jeanne s'est donc allumé dans les esprits bien avant 1431, non que je veuille assimiler les chanoines d'Orléans à la Pucelle : nous sommes dans des circonstances, avec des mentalités et au sein de groupes sociaux bien différents. Cependant de l'un à l'autre épisode, les récits qui les rapportent montrent des attitudes proches que l'historien peut tenter de discerner et d'expliquer. Ce sont les récits des chroniqueurs et historiens du $\mathrm{XV}^{\mathrm{e}}$ siècle qui ont été choisis comme fils conducteurs, ils vont nous permettre d'approcher le sens que l'on donnait avant 1480 au feu allumé le 30 mai 1431 sur la place du vieux marché de Rouen. Le corpus choisi, a été par commodité, et malgré toutes leurs menues imperfections, largement puisé dans les tomes IV et V de Quicherat ${ }^{11}$ qui demeurent encore irremplacés.

Du coté des partisans du roi Charles VII. le cas extrême est celui du héraut Berry. Après la prise de la Pucelle à Compiègne, celle-ci disparaît totalement de la Chronique, pourtant renseignée au plus près du pouvoir ${ }^{12}$. Les lecteurs étaient sans doute censés savoir comment s'était terminée la capture. On sait que faire de l'histoire est trier dans les faits, on peut quand mème rester perplexe quant aux motifs de ce silence. Il est possible d'y lire l'embarras d'un partisan royal à la fois à propos de l'incapacité royale à reprendre la Pucelle soit par la force, soit en l'achetant, et à l'égard des doutes que l'entourage du roi n'avait cessé de manifester sur l'origine de l'inspiration même de Jeanne ${ }^{13}$. La Chronique qui se poursuit jusqu'en 1457 ne peut pas évoquer l'annulation d'un procès dont elle s'était bien gardée de parler ${ }^{14}$. Berry n'est d'ailleurs pas le seul à jeter le voile sur la sentence du 7 juillet 1456, nous constaterons que Jean Chartier fit de même.

${ }^{10} \mathrm{Mgr}$. Drouais, l'Inquisition, Paris, Plon. 1906. ch. XIV. L'auteur essaya de montrer qu'en dépit des apparences "l'Eglise ne porte en rien la responsabilité de l'issue suprême », en s'appuyant sur le fait que le jugement de l'Eglise était suivi d'un jugement séculier.

11 J. Quicherat, Les Procès de Jeanne d'Arc, Témoignages des chroniqueurs et des historiens $d u X V$ siècle, Paris, (Société de l'histoire de France). 1847, t.IV; 1849, t.V.

${ }^{12}$ Les chroniques du roi Charles VII par Charles le Bouvier dit le Héraut Berry, éd. H. Courtault et L. Célier, avec la collaboration de Marie-Henriette Jullien de Pomerol, Paris, (Société de l'histoire de France), 1979, p. 144 : durant lequel siege [de Compiègne] fut prinse la Pucelle d'un Picart qui depuis la vendit à messire Jehan de Luxembourg et aux Englois.

${ }^{13} \mathrm{Ph}$. Contamine, "Mythe et histoire: Jeanne d'Arc. 1429 », Mythes et Histoire, Razo, 12, 1992, pp. 41-54: «On a souvent relevé la prudence. voire la méfiance avc laquelle la monarchie des Valois a accueilli le message johannique » (p. 51).

${ }_{14}$ La Chronique de la Pucelle, èd. Vallet de Viriville. Paris. 1859, s'arrête brusquement après l'épisode de la porte Saint-Honoré et n'aborde pas les difficultés et la mort de Jeanne. Il 
La responsabilité conjointe des clercs et des Anglais n'est évidente et le type de supplice de Jeanne ne fut fixé par leur accord préalable que pour Perceval de Cagny, mais une regrettable lacune du texte ne permet de citer que Louis de Luxembourg parmi les ecclésiastiques complices: « le dit de Bethfort et les dessus dits nommez la tiendrent en leurs prinsons oudit lieu de Rouen [...], desirans, de trouver à leur pouvoir en et sur elle une maniere d'eresie [...] Et à la parfin jetèrent leurs sentences et par eulx fut condampnée et jugée à être arsse $»^{15}$. L'exemplarité voulue de la peine est soulignée par le chroniqueur qui, dans un paragraphe spécial «Quand la Pucelle fut arsse» précise que l'exécution prit place « en lieu qui peult estre veu de tres grant peuple ». Nous retrouvons la mise en scène solennelle, le lieu «apareillé » et le feu prêt, utilisés déjà par le roi Robert pour prouver la vertu d'anéantissement de l'exécution. Cagny cependant ne fait aucun commentaire sur l'attitude et les sentiments des spectateurs, et à dire vrai ne laisse transparaître aucun engagement personnel.

Pour les autres chroniqueurs armagnacs, les responsables sont les seuls Anglais. Jean Chartier, qui peut-être à partir de 1420-1422 travailla à Saint-Denis, donne une version des plus ambiguës : "Luxembourg la vendit aux Anglois, qui la menèrent à Rouen [..] Et après grant délation de temps, sans procès (sic!), mais de leur volenté indeue, la firent publicquement ardoir en ycelle ville de Rouen ; qui feut bien inhumainement fait, veu la vie et gouvernement dont elle vivoit, car elle se confessoit et recepvoit le corps de Nostre Seigneur par chacune sepmaine, comme bonne catholique ${ }^{16}$. L'Église est donc écartée de la responsabilité de la mort de Jeanne, tandis que la forme même de cette mort. par le feu, peine des hérétique, est directement critiquée par l'affirmation de la parfaite catholicité de la jeune fille. Chartier sous-entend que s'il y avait eu procès il eût été impossible de dresser le bûcher. La mort de Jeanne fut délibérément voulue et n'avait aucune justification. C'est jouer sur les mots, car s'il est vrai que la justice séculière ne se donna pas le temps de prononcer sa propre sentence ${ }^{17}$, le procès ecclésiastique s'était bel et bien et longuement déroulé, une condamnation avait été prononcée et la peine qui en découlait ne pouvait qu'être celle du feu. Son argumentation amena le chroniqueur à ne pas pouvoir parler de l'annulation de la sentence d'un procès qui n'aurait pas eu lieu. Or il devait certainement avoir eu connaissance de l'entreprise de réhabilitation

\footnotetext{
s'agit, en dépit de son intérêt réel, d'une oeuvre tronquée. trafiquée, qui en tout état de cause ne peut servir ici.

${ }^{15}$ Quicherat, t. IV, p. 36, et Chroniques de Perceval de Cagny, éd. H. De Moranvillé, Paris, (Société de l'histoire de France), 1902, p. 179 : " en l'an M CCCC XXXXI, le XXIL5 jour du mois de may, le duc de Bethford, l'evesque de Terouenne et plusieurs autres ".

${ }^{16}$ Quicherat, t. IV, p. 93.

17 «Elle fut remise aux mains du bailli et sans que le bailli ou moi-même à qui il appartenait de prononcer la sentence en eussions prononcé une. le bourreau sans plus prit Jeanne et la conduisit à l'endroit ou le bois était préparé et elle fut brûlée ", témoignage de Laurent Guesdon, lieutenant du bailli de Rouen (ProcËs en nullité de la condamnation de Jeanne d'Arc, éd. P. Duparc, Paris, 5 vol., (Société d'histoire de France), 1977-1988, t. I, p. 458 (texte latin).
} 
puisqu'il ne mourut qu'en 1464. De plus, son homonyme Guillaume Chartier, évêque de Paris, avait été un des acteurs importants de la procédure.

On retrouve, bien sûr, dans les Vigiles du roi Charles VII de Martial d'Auvergne, texte tardif daté entre 1477 et 1483 et écho versifié du texte précédent, cette même affirmation que seuls les Anglais portent le poids du bûcher :
A Rouen en emenèrent
La Pucelle pour prisonnière,
Elle estoit tres douce, amyable
Moutonne, sans orgueil, n'envye
Souvent elle se confessoit
Pour avoir Dieu en protecteur
$\mathrm{Ne}$ gueres festes ne passoit
Que ne reçeus son Createur.
Après plusieurs griefz et exces
Inferez en maintes parties.
Lui firent ung tel quel procès
Dont les juges estoient parties .
Puis au dernier la condamnèrent
A mourir douloureusement
Et brief l'ardirent et brullerent
A Rouen tout publicquement ${ }^{18}$.

Admirons chez Chartier comme chez le versificateur la façon astucieuse dont ne sont pas évoqués les ecclésiastiques, dont l’impartialité de la justice est mise en doute comme «juge et partie ". On note ensuite l'adresse avec laquelle l'iniquité de la douloureuse condamnation au feu est soulignée par le rappel des pratiques orthodoxes hebdomadaires d'une Pucelle "bonne catholique », dont on ne nous dit pas qu'elle ne put pas les suivre pendant le temps où elle fut prisonnière puisque ses juges lui refusèrent obstinément et presque jusqu'au bout le secours des sacrements. Le vers «Ne guere feste ne passoit... " est justement en contradiction avec les critiques de ses juges qui voulurent pousser Jeanne à avouer un usage excessif de la confession et de la communion, autre preuve de son commerce démoniaque. Quant à la brebis blanche et douce du poète, où l'on voit poindre la légende de la bergère, elle est au féminin l'agneau sacrifié. Bien que Martial d'Auvergne donne le résultat du procès en annulation dont il vante - lui - les évéques et archevêques Chartier et Jouvenel «gens du bien », par lesquels,

Fut dit, par sentence autenctique,

Le procès et le jugement

Faict contre la Pucelle inique..., ${ }^{19}$

il avait pourtant réservé son avis sur la fin cruelle de Jeanne:

${ }^{18}$ Quicherat, t.V, pp.75-76.

19 Idem, p. 77. 
Si firent mal ou autrement,

Il s'en faut à Dieu rapporter

Qui de telz cas peut seulement

Lassus congnoistre et discuter.

L'Ecossais Walter Bower, a fait reposer le poids du jugement et de la décision sur les seules épaules du duc de Bedford: "là, (à Rouen), par le jugement du seigneur Jean, régent, elle fut brûlée ${ }^{20}$. Un autre étranger, favorable aussi aux thèses des partisans de Charles, Buonincontro(†1458), épousa une opinion fort proche, peut-être celle que Charles tenait à faire répandre dans les cours étrangères, en affirmant sans nuances « [qu'] à Rouen, par le duc de Betford, elle fut brûlée »" .

Le registre delphinal de Mathieu Thomassin ( $\dagger 1456$ ), réfute la mort par le feu comme peine correspondant aux crimes dont Jeanne fut accusée. Il sait et affirme qu'il y eut procès. Qui le mena, il se garde de le préciser, qu'il fût inique, il en est certain : «et fut menée à Rouen, et là luy fut fait un procez de sa vie, pour trouver aucune chose pour la faire mourir ${ }^{22}$. Donc, il dénonce un jugement préparé d'avance, procès sans porte de sortie pour la Pucelle, car on n'avait réussi à la prendre en défaut que sur l'habit d'homme. Ce n'est pas le lieu de discuter de la validité de cette accusation et de son sens, mais pour Thomassin ce qu'on lui reprochait n'était pas de ces faute dont la punition relevait de la peine des hérétiques: "Autre chose ne sceurent trouver sur elle, mais qu'elle avoit laissé l'habit ordonné pour femme...Et nonobstant ce, elle fut condampnée à mourir au feu, pour occasion seulement dudit habit d'homme ". Ce sont les mêmes arguments, dans un style plus élégant et avec des tournures mieux frappées qu'a utilisés Piccolomini (le pape Jules II, 1458-1464) pour dire son admiration pour l'œuvre de la Pucelle (Digna res quae memoriae mandaretur), en ne parlant que des Anglais et sans laisser filtrer que les juges étaient d'honorables clerc, évêques, abbés, universitaires : « quand ils surent qu'elle avait repris les habits d'homme, les juges la condamnèrent au feu comme relapse ${ }^{23}$. L'ambiguïté est encore ici totale entre la logique interne du crime de l'hérétique relapse passible du feu et un tribunal dont le pape ne reconnaît que son caractère anglais.

On ne sait trop quelle fut l'opinion de Thomas Basin du temps qu'il vivait sous le gouvernement des Anglais - il est le contemporain exact de Jeanne, né comme elle en 1412 - , il avait dix-neuf ans en 1431 et sans aucun doute ses idées sur la question, dont il n'est pas sûr quelles aient été celles qu'il exposa ensuite dans son Histoire de Charles VII et encore moins dans le Libelle qui lui fut demandé pour le procès en annulation de la condamnation ${ }^{24}$. Basin partage les responsabilités entre

${ }^{20}$ Quicherat, t. IV, p. 480.

${ }^{21}$ Idem, p. 506 : Apud Rotomagum a duce Betford igne crematur.

22 Idem, p. 312.

${ }^{23}$ Quicherat, t. V, pp. 517-51.

${ }^{24}$ B. Guenée, Entre l'Église et l'État, quatre vies de prélats français à la fin du Moyen Age, Paris, 1987, pp. 370-371, fait une fine analyse de l'argument de la peur développée par Thomas Basin dans son Libelle pour expliquer le comportement des juges de Rouen. 
les clercs (il cite nommément Cauchon) qualifiés d' " ardents promoteurs et défenseurs du parti Anglais" et les Anglais eux-mêmes, raconte les diverses péripéties du procès, l'abjuration, la mort. Mais il affirme, donnant clairement son avis, que Jeanne fut envoyée par Dieu et que per hoc heresis, atque relapsus satis manifeste defuisse fundamenta ${ }^{25}$. La peine du feu n'était pas adéquate, mais non la mort qui est en fin de compte paradoxalement justifiée. La rhétorique de Basin vise à laisser à Dieu l'initiative de la prise de Jeanne. Citant saint Paul (Rom., XI, 34 : "qui a pu pénétrer les intentions du Seigneur? Qui a été son conseiller ? "), il admet que la Pucelle ait pu avoir été conduite à la mort à cause des "démérites du roi ou du peuple", qu'elle fut une sorte de bouc émissaire sacrifié en raison de "l'ingratitude de tant de bienfaits que Dieu leur avait envoyé merveilleusement par son intermédiaire ${ }^{26}$. Du coup, il est impossible à Basin de discourir sur la nature de la peine. On ne discute pas l'œuvre de Dieu, II ne commet jamais l'iniquité. A la limite, Basin absout ainsi les Anglais, eux-mêmes instruments de la providence divine. Celui qui avait été nommé évêque de Lisieux au temps des Anglais pouvaitil totalement les condamner?

Ainsi, au cours du siècle et même une fois le procès de Rouen frappé de nullité, le parti royal finissait-il par presque justifier, non la forme juridique de la mort, mais le sacrifice nécessaire et la réduction en cendres de Jeanne.

\section{***}

Les Anglais ou les écrivains du parti anglo-bourguignon n'ont évidemment pas suivi les mêmes raisonnements et pour la plupart le feu qui dévora la Pucelle a eu vraiment un caractère purificateur, éradicateur du mal.

Le plus lu et utilisé pendant des siècles. fut Enguerrand de Monstrelet. Comme il était attaché à la maison de Luxembourg on peut aisément comprendre qu'il ait livré dans sa Chronique (1400-1444) une vision totalement opposée à celles que nous avons évoquées jusqu'à présent. Sa méthode est également assez différente. Il utilise en la citant la lettre envoyée par les Anglais au duc de Bourgogne: une femme présomptueuse a semé le carnage au milieu des combats. $\grave{A}$ la demande du Seigneur, les Anglais sont intervenus pour défendre la Justice et incarner le courroux divin: «elle a été cause que trop grand nombre d'hommes se sont tournés vers les fables que la rumeur publique répandait dans presque tout l'univers au sujet de cette femme superstitieuse. [...] La clémence divine s'est émue de pitié à la vue de son peuple menacé, [...] pour prouver que son esprit n'était pas en elle, Dieu a mis cette misérable femme entre nos mains et en notre pouvoir ». L'apparition de Jeanne était une manifestation diabolique, sa capture un don de Dieu, l'épisode un acte du grand combat entre le Bien et le Mal. Après avoir résumé les principaux griefs contre la Pucelle, raconté l'abjuration, la lettre continuait:

25 Thomas Basin, Histoire de Charles VII, éd. Ch. Samaran, Paris, 1964 (1933), t. I, p.163: "Et ainsi l'accusation d'hérésie et de relaps manquaient clairement de base ».

${ }^{26}$ Idem, p. 165 : Quoniam non est apud Deum iniquitas, licet a nobis minime cognita ab hostibus capi et supplicio sic eam permiserit. 
« mais le feu de son orgueil qui paraissait éteint, excité de nouveau par le souffle du démon s'est rallumé en flammes pestilentielles et cette malheureuse est revenue aux erreurs et aux fables insensées qu'elle avait auparavant vomies [...] pour qu'elle ne pût infecter désormais les autres membres du Christ, elle a été abandonnée au jugement du pouvoir séculier, qui a décidé que son corps serait brûlé ». Monstrelet reprend ensuite sa propre narration, ayant au mieux utilisé la métaphore ignée filée par la chancellerie d'Henri VI : «Si fu menée par ladicte justice laye ou viel marchié dedens Rouen, et là publiquement fu arse a la vue de tout le peuple ${ }^{27}$. Le chroniqueur reprend à son compte l'idée de l'hérésiarque se brûlant lui-même du feu de son erreur, image de l'autodestruction par une sorte autodafé suicidaire permis, voire provoqué, par l'abandon divin d'une créature égarée. La leçon donnée aux spectateurs était semblable à celle offerte aux Orléanais de 1022. Pour Monstrelet, mais en suivant un tout autre raisonnement que celui suivi par Basin, ce sont bien les Anglais qui ont ainsi sauvé la Chrétienté.

Jean Nider s'inspire pareillement, dans son Formicarius rédigé en 1439, à la fois de la lettre envoyée par les Anglais à l'empereur Sigismond et des récits qui couraient dans les ambassades. Il distingue les Anglais des juges, parle des inquisiteurs français. Le procès, sous l'autorité de litteratissimorum virorum, lui semble avoir clairement établi que le commerce que la Pucelle disait avoir avec les anges était diabolique. Quant à la mort, elle fut confiée par les juges au pouvoir séculier, "public ": ipsam [Jeanne] ignibus per publicam justiciam consumi permiserunt. La juste alliance des deux pouvoirs est donc clairement énoncée, le bûcher était la seule issue ${ }^{28}$. Nider rappelle le sort identique, sans citer son nom, de Pierronne la Bretonne laquelle in pertinacia permanens, ignibus consumpta est. Les deux feux de Rouen et de Paris se justifiaient l'un l'autre et confortaient l'idée qu'ils furent indispensables pour conjurer une dangereuse épidémie de sataniques séductions auprès des femmes.

Clément de Fauquemberque $(\dagger 1438)$, greffier au Parlement de Paris, avait pu se procurer le texte d'une pancarte posée devant l'échafaud qu'il inséra dans ses registres: "Jeanne [...] menteresse, abuseresse de peuple, divineresse, cruelle, dissolue, invocateresse de déables, apostate, scismatique, hérétique », tous crimes qui relevaient bien du feu qui consuma Gomorrhe. En juriste civil, il a laissé toute la plénitude de la décision à la responsabilité des gens d'église, et a justifié leur tribunal : "et prononça la sentence messire Pierre Cauchon, évêque de Beauvais ou dyocèse duquel ladite Jeanne avoit estée prinse ${ }^{20}$. Il a rapporté que l'évêque s'était entouré de clercs de Normandie, de Paris, de théologiens et de juristes. Nul doute, les crimes étant avérés et affichés, la sentence fut juste, le supplice approprié,

\footnotetext{
${ }^{27}$ Les passages de la lettre de Bedford ne sont pas retranscrits dans le texte de Monstrelet tel que le donne J. Quicherat, t. IV, p. 403, mais le texte de la lettre avait été publié au t. I,p. 489. ${ }_{28}$ Quicherat, t. IV, p. 504. : tandem ipsa fassa est se habere familiarem Dei angelum, qui judicio litteratissimorum virorum judicatus estesse malignus spiritus ex multis conjecturis et probationibus...

${ }^{29}$ Idem, pp. 459-460.
} 
l'application efficace: Jeanne qui a été «arse et brûlée » méritait ce double anéantissement .

Le récit le plus circonstancié des chroniqueurs hostiles à la cause de Jeanne est celui du Bourgeois de Paris dans son Journal. Il connaissait les différents points débattus lors du procès - ces précisions sont une des preuves éventuelles de son appartenance à l'Université de Paris - et il en a donné un résumé à chaque fois tendancieux : «La dame Jeanne [...] a trompé le peuple, a fait idolâtrer le simple peuple, car par sa fausse hypocrisie, ils la suivaient comme sainte pucelle ${ }^{30}$. Le Bourgeois fut aussi le seul à expliquer clairement la façon dont on fit pression sur Jeanne pour la faire abjurer: "elle serait devant tout le peuple arse et son âme damnée au fons d'enfer ${ }^{31}$. Pour ma part je pense que le contexte de la peine contre les maléfices et l'hérésie permet de lire dans cette formule la redondance du «feu de l'enfer » de certaines versions, le feu terrestre consumerait le corps, le feu spirituel tourmenterait l'âme éternellement. Le Bourgeois est d'ailleurs le seul auteur contemporain à penser à l'âme immortelle de Jeanne et à la distinguer d'un corps physique réduit définitivement en cendre.

La description, par ouï-dire, du Bourgeois de Paris donne des derniers instants de la Pucelle une vision atroce et curieusement plus ouverte vers l'espérance que toutes celles que l'on a pu évoquer jusqu'à présent. À la différence de Thomas Basin, l'auteur ne doit pas avoir eu connaissance de la procédure d'annulation car son récit s'arrête en 1449 à la veille de la reprise de Rouen, cependant le supplice ne clôt pas vraiment l'histoire de Jeanne. Liée à un pieu la jeune fille fut asphyxiée (" estainte » dit-il) par la fumée, sa robe consumée permit de voir son corps nu, ses seins, son sexe, que l'on montra au peuple pour qu'on sache bien qu'elle était femme avec «tous les secrets qui peuvent ou doivent être en femme». Le feu ainsi est révélateur de la vérité, il ôte les doutes sur la personne physique de Jeanne, mais aussi la montre endurcie dans son erreur ${ }^{32}$. Ensuite on fit repartir les flammes pour que tout soit réduit en cendre, rappelons nous les pauvres restes des chanoines orléanais, eux aussi visibles dans les flammes et eux inutilement repentants avant d'être totalement mis en poudre par la combustion.

Mais contrairement à tous les autres, le Bourgeois n'arrête pas son récit. Cet homme qui jamais n'a été favorable à la Pucelle n'est pas pleinement satisfait, n'est pas complètement convaincu que la peine ait sanctionné de véritables errements. Il

30 Joumal d'un bourgeois de Paris, èd. Colette Beaune, Paris, 1990, p. 292 . La modernisation du texte est de l'éditrice.

${ }^{31}$ Idem, p. 296. C. Beaune donne en note les deux leçons possibles, feu ou fond.

${ }^{32}$ En réalité, et sans entrer dans l'inépuisable controverse des vraies et fausses morts de Jeanne d'Arc (cf. Y. Grandeau, Jeanne insultée, Procès en diffamation, Paris, 1973), nous avons ici l'écho de ce que rapporte également une Chronique Normande "Mais finalement la firent ardre publiquement, ou aultre femme en semblable d'elle ; de quoy moult de gens ont esté et sont encore de diverses oppinions ». Chronique Normande éd. Vallet de Viriville, Chronique de Charles VII par Jean Chartier, Paris, 1858, t. III, pp.106-107. Les discussions allaient déjà bon train sur la réalité de la mort, sur la personne de Jeanne, et sur la valeur de la condamnation. 
laisse parler le peuple et par la vox populi « dit». lui aussi, le doute qui subsiste: "Assez avait là et ailleurs qui disaient qu'elle était martyre et pour son droit Seigneur, autres disaient que non et que mal avait fait qui l'avait tant gardée. Ainsi disait le peuple, mais quelle mauvaiseté ou bonté qu'elle eut faite, elle fut arse celui jour ${ }^{33}$.

Le Bourgeois n'ose choisir, "passez outre" semble-t-il murmurer selon la formule que Jeanne elle-même utilisait pour ne pas répondre aux questions qui relevaient de son monde intérieur. Mais cependant le choix était fondamental : pour la quasi-totalité des historiens et chroniqueurs de son temps le bûcher de Jeanne fut celui de l'anéantissement. Le sens de la justification du brasier (purifier le roi et la France ou dévorer le Mal) et l'identification des porteurs de brandon (juges ecclésiastiques et Anglais ensembles ou séparément) marquaient seulement la différence entre les camps politiques qui déchiraient le royaume de France. Seul le Bourgeois de Paris par ses doutes ouvrait, innocemment mais très tôt, la voie à un sens nouveau du rituel judiciaire de Rouen, celui d'un feu démiurge, qui au lieu de réduire la Pucelle en cendres à jamais perdues la faisait naître à une autre vie, celle des martyrs, au rang de ceux dont la place était non pas dans la Géhenne ardente, mais à l'intérieur du cercle de feu des chérubins, dans la chaleur rayonnante de la contemplation de Dieu.

Françoise Michaud-Fréjaville

${ }^{33}$ Journal d'un Bourgeois de Paris, p. 297. On voit que le Bourgeois n'a pas, pour sa part, de doute sur la mort effective de Jeanne d'Arc à Rouen. 